\title{
Ziel: Möglichst unauffällige Narbe
}

Für einen möglichst narbenfreien Wundverschluss benötigt es neben der Wahl des passenden Verfahrens auch große chirurgische Erfahrung. Die Möglichkeiten, eine Wunde zu schließen, reichen von Nahtmaterial, Klammern bis zum Klebstoff.

Fortschritte im Bereich des Wundverschlusses bildeten einen der Schwerpunkte des diesjährigen Deutschen Chirurgenkongresses im vergangenen Frühjahr in München.

„Die Hautnaht ist die Handschrift des plastischen Chirurgen - die bleibende, möglichst dezente Erinnerung an eine gute Operation", betonte Prof. Dr. Peter M. Vogt, Präsident der Deutschen Gesellschaft für Chirurgie (DGCH) und plastischer Chirurg. Selbstauflösende Fäden haben den Vorteil, dass ein schmerzhaftes Fädenziehen ent- fällt - sie sind daher besonders für Kinder geeignet. Ihre Reißfestigkeit ist jedoch gegenüber nicht-resorbierbaren Fäden begrenzt.

Alternativen für die Grundlage von möglichst unauffälligen Narben sind Klammern und Wundkleber. Klammern aus Edelstahl werden mit speziellen Geräten ins Gewebe gedrückt und nach etwa zehn Tagen wieder entfernt. Sie kommen bevorzugt im behaarten Kopfbereich zur Schonung der Haarfollikel zum Einsatz. Das Ergebnis sind sehr feine Narben. Bei kleineren Wun- den kommen auch Wundkleber in Frage. "Cyanoacrylate" werden zum Verschluss oberflächlicher Wunden im Gesicht, besonders bei Kindern genutzt. Mitunter können sogar kleine Klebestreifen ausreichen.

Quelle:

Presseaussendung DGCH

Wien klin Mag $2015 \cdot$ 18:183

DOI 10.1007/s00740-015-0072-3

Online publiziert: 8. Oktober 2015

(c) Springer-Verlag Wien 2015 